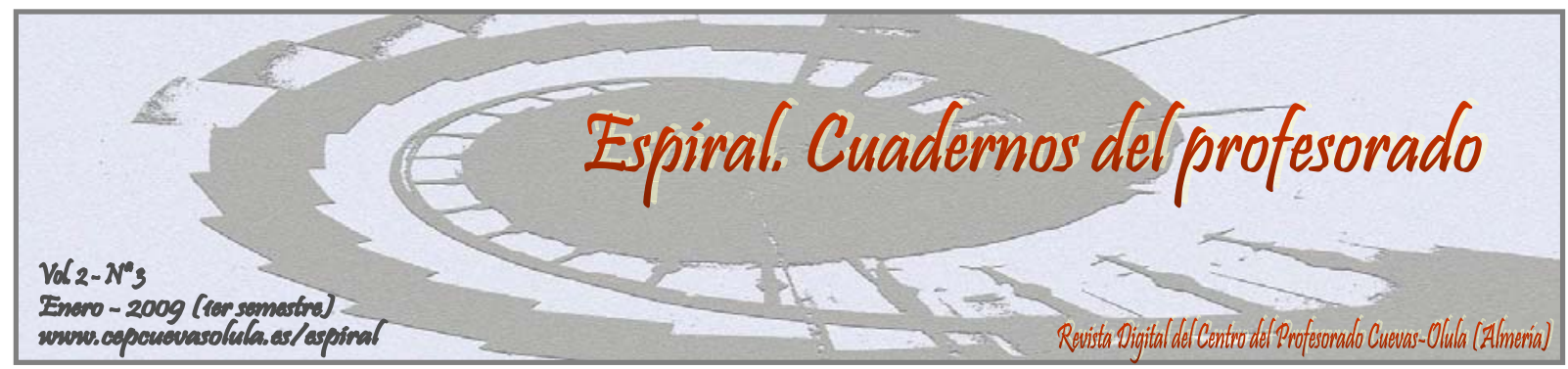

UNA EXPERIENCIA DIDÁCTICA EN METODOLOGÍA INTEGRADA: CINCO ESTILOS DE ENSEÑANZA A TRAVÉS DE LA BICICLETA DE MONTAÑA, PATINES Y MONOPATINES EN E.S.O.

\author{
TEACHING EXPERIENCE IN AN INTEGRATED METHODOLOGY: FIVE \\ STYLES OF TEACHING THROUGH THE MOUNTAIN BIKE, SKETES AND \\ SKATEBOARD IN HIGH SCOOLH
}

\author{
Antonio Baena Extremera ${ }^{(1)}$, Juan Francisco Calvo Morales ${ }^{(2)}$ \\ y Marina Martínez Molina ${ }^{(1)}$

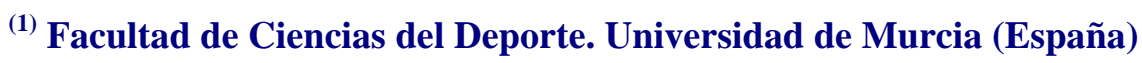 \\ ${ }^{(2)}$ I.E.S. Martín García Ramos, Albox, Almería (España)
}

Baena Extremera, A., Calvo Morales, J. F. \& Martínez Molina, M. (2009). Una experiencia didáctica en metodología integrada: cinco estilos de enseñanza a través de la bicicleta de montaña, patines y monopatines en E.S.O. [en línea]. Espiral. Cuadernos del Profesorado, 2(3), 41-48. Disponible en: http://www.cepcuevasolula.es/espiral.

Enviar correspondencia a: abaenaextrem@yahoo.es

RESUMEN: El objetivo principal de esta experiencia, es mostrar una práctica docente en la cual, para los contenidos de Coordinación y Equilibrio, se ha trabajado con la Bicicleta de Montaña, los Patines y Monopatines, utilizando una metodología integrada. Para ello, se han utilizando los estilos de Enseñanza Modular, Asignación de Tareas, Grupos de Nivel, Programa Individual interrelacionados dentro de la misma sesión y como parte de una Unidad Didáctica innovadora y globalizadora de contenidos.

Palabras clave: metodología, estilos de enseñanza, unidad didáctica, bicicleta de montaña, patines y monopatines.

ABSTRACT: The main goal of this experience is to show and demonstrate a teaching activity, in which for the coordination and balance contents, the mountain bike, rollerblades and skateboard were used. The modular teaching, task assignment, level grouping and individual progam styles have been used in order to carry out such experience. Their interrelationship allow, within the same session, to form altogether a single innovative didactic-unit enclosing different contents.

Key words: methodology, teaching styles, didactic unit, mountain bike, rollerblades, skateboard. 


\section{1.- INTRODUCCIÓN.}

El presente artículo intenta explicar una experiencia realizada en el I.E.S. Cardenal Cisneros de Albox (Almería). Este trabajo, se ha llevado a cabo dentro del primer trimestre y correspondiendo a la Unidad Didáctica (UD) número 2, según las orientaciones descritas por Baena (2007) para la programación de aula, siendo esta dedicada para $1^{\circ}$ ESO. Para ello, se han empleado tres materiales diferentes, como son la Bicicleta Todo Terreno (Btt), los Patines (Pt) y los Monopatines (Mnp), con el fin de trabajar un contenido establecido desde el Decreto 231/2007, de 31 de julio, por el que se establecen las enseñanzas correspondientes a la Educación Secundaria Obligatoria en Andalucía, donde el bloque de contenidos de Juegos y Deportes incluye como contenidos relacionados con esta unidad:

- Coordinación y equilibrio en sus diferentes manifestaciones, en relación con la ejecución de habilidades motrices.

- Adquisición y refinamiento de las habilidades y dominio corporal sin elementos, con objetos y personas.

- Tareas motrices que mejoren la percepción, la estructuración espacio-temporal, la atención selectiva y la anticipación.

La idea principal de dicha UD era trabajar, estos contenidos con materiales y actividades diferentes y novedosas, puesto que lo tradicional para las sesiones de coordinación y el equilibrio no ha sido la Btt, Pt y Mnp. En la etapa anterior y según la Orden del 10 de Agosto de 2007 que establece el currículum para la Educación Primaria en Andalucía, dentro del bloque de Habilidades Motrices se ha de mejorar estas cualidades perceptivo-motrices. Atendiendo a esto, se ha programado para secundaria y según el desarrollo curricular vigente, UD para afianzar y automatizar la coordinación y el equilibrio desde la utilización de otros materiales.

Para llevar a cabo la práctica, se ha optado por utilizar diferentes estilos dentro de una misma técnica de enseñanza coordinados entre dos profesores: Enseñanza Modular, Asignación de Tareas, Grupos de Nivel, Programa Individual y Resolución de Problemas, utilizando el modelo de UD planteado por Baena (2008).

\section{2.- DESARROLLO DE LA U. D. DE METODOLOGÍA INTEGRADA.}

\section{Justificación.}

La conducta motriz está asociada a que el alumnado comprenda su propio cuerpo, constituyendo un todo que pueden encontrar en cada experiencia o vivencia que experimenten.

Atendiendo al D. 231/2007, nos encontramos que en el bloque de contenidos de Juegos y Deportes, queda reflejado que para la Educación Secundaria Obligatoria se deberán consolidar y perfeccionar las habilidades motrices vinculadas a acciones deportivas, dentro de las que debemos incluir las cualidades de coordinación y equilibrio a través de diferentes propuestas que incluyan juegos, actividades deportivas, etc.

Como en la Programación de Aula de $1^{\circ}$ ESO hay diseñada una UD de Btt para el tercer trimestre, se han introducido aspectos técnicos como relación intradisciplinar entre unidades.

La idea inicial era trabajar únicamente con la Btt, pero el problema surgía al haber alumnos que no disponían de ellas, así que se optó por usar los Pt y Mnp. La UD aparte de incluir todos los elementos del currículum, para hacerla más innovadora y globalizadora de contenidos se ha relacionado con:

- La Interdisciplinariedad según el artículo 24 y 25 de la Ley Orgánica de Educación 2/2006 del 3 de mayo.

- La Intradisciplinariedad del área, a través de los Bloques Asociados de Juegos y Deporte y Actividad Física en el Medio Natural (UD 10 de la Programación de Aula sobre la Btt).

- Con los Temas Transversales según la Orden 17 de Enero de 1996 que establece la organización y el funcionamiento de los programas sobre Educación en Valores y Temas Transversales del currículum y la Orden 19 de Diciembre de 1995 por el que se establece el desarrollo de la Educación en Valores en los centros docentes de Andalucía.

- Con la Cultura Andaluza según la Orden 6 de Junio de 1995 por la que se aprueban los objetivos y el funcionamiento del programa de Cultura Andaluza.

- Con la Atención a la Diversidad según la Orden 13 de Julio de 1994 por la que se regula el proceso de diseño, desarrollo y aplicación de adaptaciones curriculares en los centros docentes. 
La UD quedaría redactada de la siguiente forma (tabla 1):

Tabla 1: Desarrollo de la Unidad Didáctica.

\begin{tabular}{|l}
\hline $\begin{array}{l}\text { Centro de Interés: Nos desplazamos, coordinados y sin caernos } \\
\mathbf{N}^{\circ} \text { Alumnos: } 60 \quad \text { Trimestre: } 1^{\circ}\end{array}$ Sesiones: 5 \\
\hline \multicolumn{4}{c}{ OBJETIVOS DE LA UNIDAD DIDÁCTICA $1^{\circ} \mathrm{ESO}$ A-C } \\
- Mejorar la coordinación segmentaria y la coordinación dinámica general, así como el equilibrio estático \\
y dinámico. \\
- Practicar ejercicios de coordinación y equilibrio con implementos. \\
- Mejorar y afianzar las cualidades perceptivo-motrices. \\
- Realizar un circuito de evaluación inicial y final. \\
- Adquirir conocimientos técnicos básicos sobre el uso de la Btt, Pt y Mnp. \\
- Fomentar el uso de la Btt, los Pt y Mnp en el tiempo libre. \\
- Valorar y respetar el propio nivel de coordinación y equilibrio, el de los compañeros, así como las \\
instalaciones, equipamientos y material.
\end{tabular}

\begin{tabular}{|l|c|c|c|c|c|c|c|c|c|c|c|c|c|c|}
\hline Relación con O. Etapa & $\mathrm{A}$ & $\mathrm{B}$ & $\mathrm{C}$ & $\mathrm{D}$ & $\mathrm{E}$ & $\mathrm{F}$ & $\mathrm{G}$ & $\mathrm{H}$ & $\mathrm{I}$ & $\mathrm{J}$ & $\mathrm{K}$ & $\mathrm{L}$ & $\mathrm{M}$ & $\mathrm{N}$ \\
\hline Relación con O. Área & \multicolumn{1}{|c|}{1} & \multicolumn{2}{|c|}{2} & 3 & 4 & & 5 & & 6 & 7 \\
\hline
\end{tabular}

- Bloque organizador: Cualidades Motrices

- Bloques asociados: Juegos y Deportes, Actividad Física en el Medio Natural

\begin{tabular}{l|l|}
\multicolumn{1}{c|}{ CONCEPTOS } & \multicolumn{1}{|c|}{ PROCEDIMIENTOS } \\
- Coordinación segmentaria y & - Realización de un circuito de \\
dinámico-general & evaluación inicial y final. \\
- Equilibrio estático y dinámico & \multicolumn{1}{c}{ Practica, mejora y } \\
- Btt. & afianzamiento de la coordinación \\
- Pt. & y el equilibrio sobre otros \\
- Mnp. & implementos. \\
& - Realización de juegos y tareas \\
& de coordinación y equilibrio con \\
& la Btt, Pt y Mnp.
\end{tabular}

\begin{tabular}{l}
\multicolumn{1}{c}{ ACTITUDES } \\
- Aceptación de las diferencias y \\
posibilidades de cada uno. \\
- Actitud positiva hacia la tarea. \\
- Valoración de las múltiples \\
alternativas que nos ofrecen la \\
Btt, Pt y Mnp para organizar el \\
tiempo libre.
\end{tabular}
tiempo libre. de coordinación y equilibrio con METODLOGÍA

\begin{tabular}{|c|c|c|}
\hline \multicolumn{3}{|c|}{ METODLOGÍA } \\
\hline \multicolumn{2}{|c|}{ INSTRUCCIÓN DIRECTA } & INDAGACIÓN O BÚSQUEDA \\
\hline $\begin{array}{l}\text { - Asignación de Tareas } \\
\text { - Grupos de Nivel } \\
\text { - Enseñanza Modular } \\
\text { - Programa Individual. }\end{array}$ & \multicolumn{2}{|c|}{ - Resolución de Problemas. } \\
\hline \multicolumn{3}{|c|}{$\begin{array}{l}\text { - Estrategia en la Práctica: Analítica secuencial y Global polarizando la atención. } \\
\text { - Organización de los alumnos: Calentamiento (masiva), Parte Principal (parejas y gran grupo) y Parte } \\
\text { Final (masiva y gran grupo). } \\
\text { - Ppos. Metodológicos: Tendremos en cuenta para el desarrollo de la UD, los principios } \\
\text { Psicopedagógicos y Didácticos descritos desde el D.C.B., como son: 1) Partir del nivel del alumno y de } \\
\text { su experiencia; 2) Potenciar el aprendizaje significativo; 3) Adaptar los métodos y recursos a las } \\
\text { peculiaridades de cada grupo y alumno; 4) Aprender a aprender y memorización comprensiva,... }\end{array}$} \\
\hline \multicolumn{3}{|c|}{ EVALUACIÓN } \\
\hline \multicolumn{3}{|c|}{ INSTRUMENTOS Y PROCEDIMIENTOS } \\
\hline \multicolumn{3}{|c|}{$\begin{array}{l}\text { - Profesor: Diario del profesor y encuesta a los alumnos. } \\
\text { - Alumnos: }\end{array}$} \\
\hline CONCEPTOS & PROCEDIMIENTOS & ACTITUDES \\
\hline $\begin{array}{l}\text { - Cuaderno del alumno/ diario } \\
\text { - Preguntas orales sobre los } \\
\text { conceptos. }\end{array}$ & $\begin{array}{l}\text { - Seguimiento de la ejecución } \\
\text { correcta a través de la } \\
\text { Observación Sistemática. } \\
\text { - Participación activa de los } \\
\text { alumnos en las clases. } \\
\text { - Prueba práctica en un circuito } \\
\text { de evaluación. }\end{array}$ & $\begin{array}{l}\text { - Registro sistemático de la } \\
\text { actitud del alumno (completa } \\
\text { tareas y se interesa, relación con } \\
\text { otros alumnos y con el profesor) } \\
\text { a través de una Lista de Control. }\end{array}$ \\
\hline
\end{tabular}


- Mejora la coordinación y equilibrio.

\section{CRITERIOS DE EVALUACIÓN}

- Practica ejercicios de coordinación y equilibrio con implementos.

- Afianza las cualidades perceptivo-motrices.

- Valora y respeta el propio nivel de coordinación y equilibrio, el de los compañeros, así como las instalaciones, equipamientos y material.

\section{- Calificación:}

30\% Conceptos (cuaderno 15\%+ preguntas 15\%).

50\% Procedimientos (seguimiento 20\%+ participación 10\%+ prueba 20\%).

20\% Actitudes (registro 20\%)

- Recuperación: Conceptos (trabajo de síntesis). Procedimientos (contrato profesor/alumno, práctica en casa y nueva evaluación). Actitudes (registro en UD posterior).

- Evaluación UD: Hoja de reflexión sistemática del docente.

\section{RECURSOS DIDÁCTICOS}

- Material: Colchonetas, bancos suecos, aros, conos, Btt, Pt y Mnp.

- Otros documentos/materiales de apoyo a sesión: Hoja de Observación Sistemática, Hoja de registro para evaluar los circuitos. Lista de Control de Actitudes.

- Instalación: Gimnasio, pistas polideportivas y superficie de tierra.

\section{INTERDISCIPLINARIEDAD}

a) CC.NN.: Circuitos en la naturaleza, uso de la BTT como medio de transporte.

b) CC.SS, $\mathbf{G}^{\mathrm{a}}$ e $\mathbf{H}^{\mathrm{a}}$ : Origen e Historia de la Btt, Pt y Mnp.

c) Ed. Plástica y Visual: Construcción de circuito de habilidad. Memoria Visual de los recorridos.

d) Lenguas extranjeras: Skate, Mountan Bike. e) Matemáticas y Física: Distancias, trayectorias y velocidades.

g) Lengua Castellana: nombre de los aspectos técnicos (derrapaje...). Nombres específicos de las partes de la Btt (potencia, pedales, ...)

h) Tecnología: Materiales, mecánica.

\section{CULTURA ANDALUZA}

- Vamos a relacionar esta UD con la Cultura Andaluza aportándole al alumnado en la sesión 1 un listado de zonas con lugares de interés medioambiental de la provincia de Almería para ir con la Btt.

\section{ATENCIÓN A LA DIVERSIDAD}

- Para atender al alumno con necesidad educativa temporal (lesionado), se va a elaborar un Programa Individual para él.

\section{TEMAS TRANSVERSALES}

Ed. Ambiental: Limpieza, orden y mantenimiento en buen estado de las instalaciones. Uso de la Btt, Pt y Mnp como medios de transporte y paseo ecológicos.

- Ed. Paz: Juegos de cooperación, respeto a los compañeros.

- Ed. del Consumidor: Valorar la Btt, el Pt y Mnp como alternativa al tiempo libre. Incentivar otros deportes menos demandados.

- Ed. Vial: Mostrar las posibilidades de ocio y recreación de la ciudad. Respeto a las normas y uso de los juegos de Btt, Pt y Mn. Circulación Vial.

- Ed. Salud: Despertar interés y gusto por el cuidado del cuerpo tras la sesión. Uso de la Btt para realizar actividad física para la salud.

- Ed. para Igualdad: Utilización de grupos mixtos e igualdad de oportunidades en las actividades.

- Ed. Sexual: Ver las diferencias sexuales de forma natural y sin discriminación sexual.

- Ed. Moral y Cívica: Respeto a las normas, respeto a los compañeros, tolerancia, autonomía en las actividades, cuidado de los materiales.

\section{PREVISIÓN DE CLASE}

SESIÓN 1: Evaluación Inicial a través de un circuito.

SESIÓN 2: Coordinación y Equilibrio I.

SESIÓN 3: Coordinación y Equilibrio II.

SESIÓN 4: Coordinación y Equilibrio III.

SESIÓN 5: Evaluación Final a través de un circuito.

\section{Del alumno:}

\section{BIBLIOGRAFÍA}

- Apuntes proporcionados por el Docente sobre la Coordinación y el Equilibrio.

- Apuntes proporcionados por el Docente sobre la Historia y Origen de la Btt, Pt y Mnp.

- Hoja de lugares de interés medioambiental de la Provincia de Almería para utilizar la Btt.

Del profesor:

- Artó Polanco, Y. y cols (1992). La E. F. en Secundaria. Su práctica. Edit. CEPID.

- Baena, A. (2005): Actualización científico-didáctica de la E. F. escolar. Granada: Gioconda.

- Baena, A. (2005): Aplicaciones Didáctica del currículo para el área de E. F. Granada: Gioconda. 


\section{Desarrollo de las sesiones.}

A modo de ejemplo, vamos a desarrollar la sesión 2, para comentar el trabajo realizado con los estilos de enseñanza dentro de la misma sesión.

Curso: $1^{\circ}$ ESO A-C

UD: Nos desplazamos, coordinados y sin caernos Sesión: $2 / 5$

$\mathbf{N}^{0}$ Alumnos: 60

\section{Descripción de los elementos curriculares.}

Objetivos de la sesión:

1. Conocer la historia y el origen de la Btt, $\mathrm{Pt}$ y Mnp.

2. Participar en juegos y ejercicios para la mejora de la coordinación óculosegmentaria y el equilibrio estático y dinámico.

3. Valorar y respetar tus posibilidades, las de los demás, así como los materiales y las instalaciones.

Contenidos de la sesión:

$\square$ Conceptuales:

- Concepto de coordinación y tipos.

- Concepto de equilibrio y tipos.

- Historia y origen de la Btt, Pt y Mnp.

$\square$ Procedimentales:

- Práctica y mejora de la coordinación y el equilibrio a través de juegos y ejercicios.

$\square$ Actitudinales:

- Respeto a los compañeros, a los demás $\mathrm{y}$ al material.

Metodología:

$\square$ Técnicas de Enseñanza y estilos:

- ID: Asignación de Tareas, Grupos de Nivel, Enseñanza Modular y Programa Individual.

Estrategias en la práctica: Global y global polarizando la atención.

$\square$ Organización de los alumnos:

- Parte Inicial: Masiva

- PP: Gran grupo de 30, 20, 10.

- VC: Gran grupo de 30, 20, 10.

Recursos didácticos:

Material: Colchonetas, bancos suecos, aros, conos, Btt, Pt y Mnp. $\square$ Otros documentos/material de apoyo de la sesión: Hoja de Asignación de Tareas, Hoja de Historia y Origen de la Btt, Pt y Mnp.

$\square$ Instalaciones Deportivas: Gimnasio, pistas polideportiva y superficie de tierra.

La primera decisión y estilo de enseñanza consistió en unificar dos clases de $1^{\circ} \mathrm{ESO}$, grupo A (30 alumnos) y C (30 alumnos), con una Enseñanza Modular, puesto que el Jueves a última hora de clase (13:25 a 14:25) coincidíamos los dos docentes de Educación Física con los dos cursos de primero. A continuación, se optó por el estilo de Grupo de Intereses, de manera que se dio a elegir entre la utilización de la Btt, Pt y Mnp según su interés y disponibilidad. Se intentó (pero no se consiguió) hacer coincidir igual número de alumnos por grupo, para poder diseñar actividades y juegos globales al final de las sesiones. Al repartir y distribuir los discentes, se estableció los grupos de la siguiente forma: Btt con 30 alumnos, Pt con 20 alumnos y Mnp con 10.

Tras la sesión 1 con la evaluación inicial, se estableció el número de grupos de nivel que se deben hacer, decidiendo proponer 2 niveles para Btt (iniciación y perfeccionamiento), 2 niveles para Pt (iniciación y perfeccionamiento) y 1 nivel para Mnp (iniciación). Como partimos de un trabajo por Grupos de Nivel, la planificación de las tareas de cada grupo las decidió el profesor y la Información Inicial fue distinta para cada subgrupo. La actuación de cada subgrupo fue diferenciada, así como el, Conocimiento de Ejecución (CE) y el Conocimiento de Resultados (CR) que fue individual y específico.

En la sesión 2, ubicamos a los patines dentro del gimnasio por haber una mejor superficie de rodaje. Esta instalación se dividió en 2 zonas, una cercana a las espalderas para el grupo de iniciación, y el resto para el grupo de perfeccionamiento.

Una de las pistas polideportivas se utilizó para la Btt de iniciación, mientras que la superficie de tierra (de una extensión de unos $1600 \mathrm{~m}^{2}$ ) fue destinada para la Btt perfeccionamiento. La otra pista polideportiva se quedó para el uso de los Monopatines.

Para cada grupo, tanto en iniciación como perfeccionamiento, se elaboró una Hoja de la Historia y Origen del material a usar y una Hoja de Asignación de Tareas, donde se especifica: posición básica, caídas y listado de tareas. Para el 
estilo de Asignación de Tareas, se partió de una organización muy bien cuidada y de una Información Inicial, de carácter general, clarificadora. La Información inicial por parte del profesor fue verbal y visual después de un análisis cuidadoso de la tarea a realizar, proporcionándola desde una posición destacada, indicando las órdenes de inicio y parada de la actividad. La organización fue masiva, con la ejecución de una lista de tareas única para todo el mundo, realizada de forma simultánea (todos a la vez). Durante la ejecución, los profesores administraron CE y CR, estimulando e interactuando con los alumnos, mientras evolucionaban entre ellos.

Con respecto a la Enseñanza Modular, se dio la posibilidad de que el alumno pudiera elegir entre diversas actividades, según sus intereses. Se ofrecieron a los alumnos dos módulos de aprendizaje, tantos como profesores y dentro de ellos se trabajo en la modalidad de niveles como hemos comentado.

Puesto que había un alumno con una necesidad educativa temporal (debido a una lesión a punto de curar) se optó por elaborar un Programa Individual de actividades y tareas específicas para él. Este estilo consiste en la elaboración por parte del docente de un programa adaptado a sus características, donde se busque la repetición de tareas similares a las trabajadas en clase, realizándose bajo el mando del profesor pero sin que él esté presente. La información se realiza a través de un programa escrito acompañado de dibujos y fotografías de las tareas. Este programa está basado en la evaluación inicial de la sesión 1, puesto que la lesión ocurrió el día posterior a la sesión primera. La evaluación del Programa Individual la realizó el docente una vez terminado el mismo.

\section{3.- REPERCUSIÓN SOBRE LA COMUNI- DAD EDUCATIVA.}

En relación a los alumnos, es una práctica divertida y distinta a lo habitual en educación física, por ello su motivación e interés fue mayor durante toda la UD y la sesión. Tienen que conocer cuantos más deportes mejor, y es nuestra labor introducirlos en todos los que podamos dentro de los recursos de los que dispongamos, con el fin de que ellos se decidan por los que más les interesen y los sigan practicando en su vida diaria.

Para que esto ocurra, tienen que ser clases adaptadas a las capacidades de los niños con las que estamos trabajando, de esta manera se verán capaces de realizarlo y querrán seguir aprendiendo, siendo siempre un aprendizaje divertido. La experiencia realizada, nos muestra una forma clara de individualizar la enseñanza, puesto que optamos por la utilización de

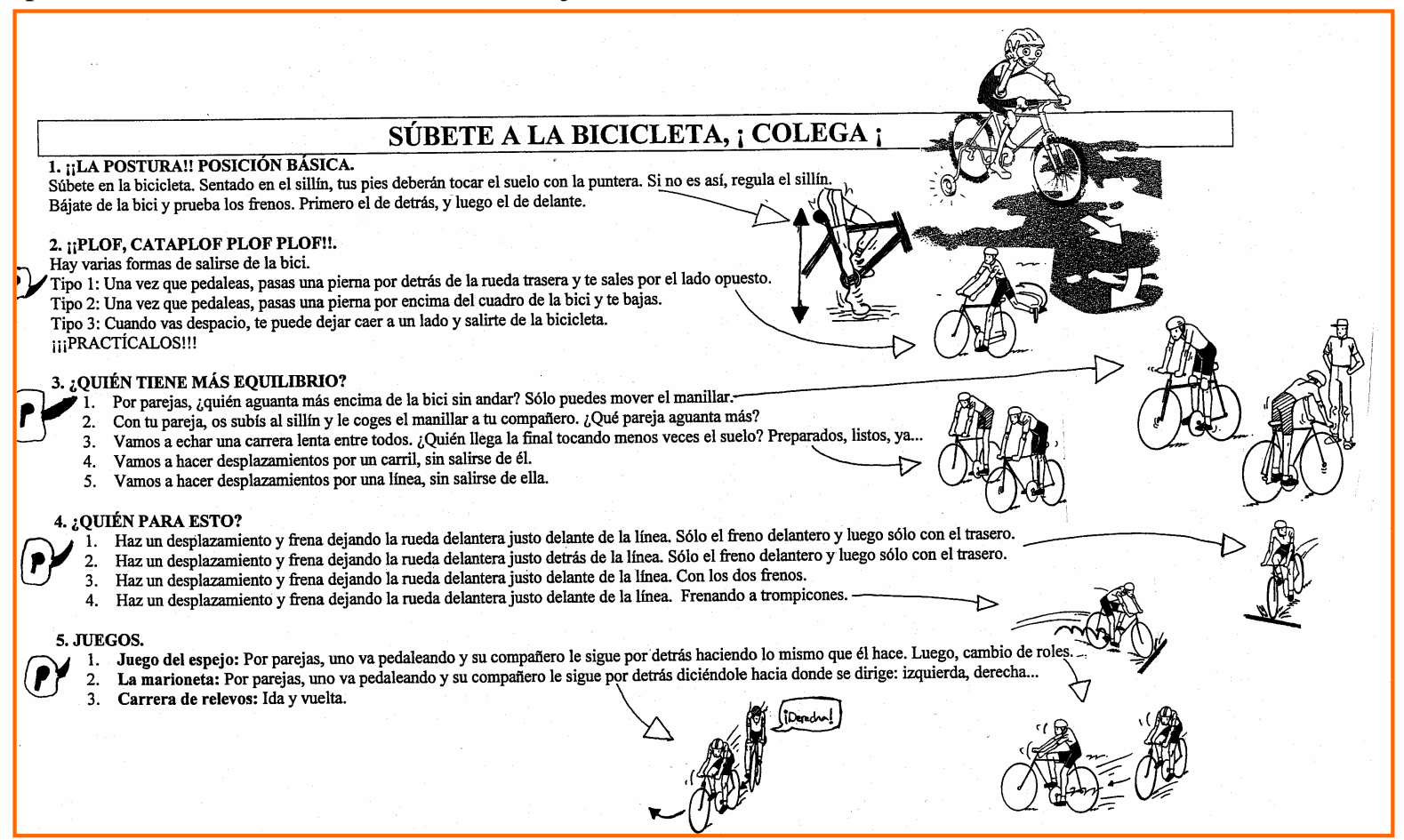

Figura 1.- Hoja de tareas 1 
diferentes estilos de enseñanza para poder atender a las necesidades de cada alumno (enseñanza modular, grupos de niveles, etc.).

Para poner en práctica esta unidad didáctica es necesaria una gran organización, ya que los centros educativos no disponen de estos materiales, y también una gran colaboración por parte de los alumnos a la hora de ceder los materiales de los que ellos dispongan. Con esta propuesta, hemos resuelto uno de los grandes problemas en la enseñanza: "el disponer de materiales iguales para todo el mundo". Como esto no era posible, hemos optado por trabajar con estos tres elementos (bicicleta, patines y monopatines).

Durante la realización de esta práctica algunos de los alumnos se iniciarán y otros seguirán aprendiendo y poniendo en práctica lo que ya saben. Pero siempre valorando y respetando tanto a sus compañeros como al material con el que están trabajando.

El trabajar con materiales innovadores dentro de un centro es una forma distinta y divertida de adquirir diversos contenidos como son: la coordinación, el equilibrio, dominio corporal, la percepción, la estructuración espacio- temporal...etc. Además de afianzar todos estos contenidos durante la práctica, también adquirirán los conocimientos técnicos básicos sobre el uso de la Btt, Pt y Mnp.

Con este artículo lo que se pretende es dar a conocer que existen otras formas muy distintas a las que estamos acostumbrados de enseñar, donde lo más importante para un buen aprendizaje es la motivación y el interés del niño, por ello se necesitan prácticas divertidas y a la misma vez educativas. Para ello se tendrá que tener en cuenta el nivel y la experiencia del alumno y adaptar, cuando sean necesarios, los métodos y recursos a las peculiaridades de cada grupo o de cada alumno.

Otro de los problemas resueltos con esta experiencia es la coincidencia de varios cursos en la misma pista polideportiva. Esto ocurre a diario en varias horas, donde dos o más docentes de

Educación Física coinciden dando clase y utilizando los mismos materiales. Con la propuesta presentada, llegamos a solventar este problema tan común en la enseñanza escolar.

La innovación educativa es además una de las mejores formas de mejorar la enseñanza. Nuestra propuesta innova pues dentro de los

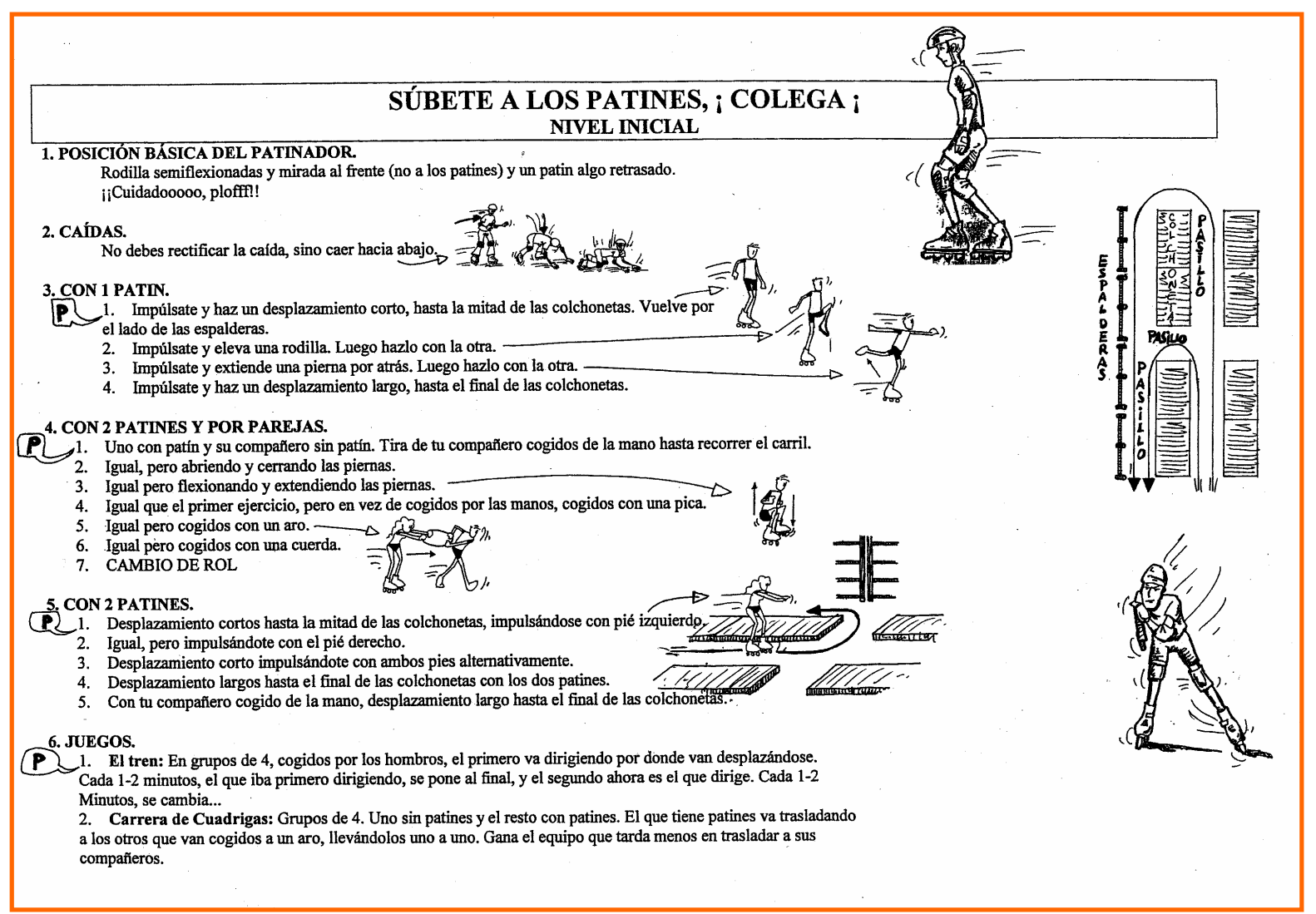

Figura 2.- Hoja de tareas 2 
estilos de enseñanza, ya que en raras ocasiones, se combinan hasta cinco estilos de enseñanza dentro de la misma sesión. Esto, ayuda y hace entender al profesorado que es la metodología la que debe estar supeditada a la sesión y no al revés.

Para terminar, ejemplificamos las dos Hojas de Tareas (figura 1 y 2) utilizadas en esta sesión, para que sirvan de ejemplo al resto de profesorado.

\section{4.- REFERENCIAS BIBLIOGRÁFICAS.}

Baena, A. (2005). Aplicaciones didácticas del currículm para el área de EF. Granada: Gioconda.

Baena, A. (2007). La programación de aula en Educación Física en secundaria: propuesta para la nueva ley orgánica de educación y para el proceso selectivo del cuerpo de profesores. Espacio y Tiempo, (53), 12-18.

Baena, A. (2008). Análisis del diseño de las unidades didácticas en educación física en relación a la etapa educativa: una propuesta innovadora. Habilidad Motriz, 30, 30-48.

Contreras, O. (1998). Didáctica de la EF. Un enfoque constructivista. Zaragoza: Inde.

Delgado, M.A. (1991). Los estilos de enseñanza en Educación Física. Granada: ICE.

Mosston, M. \& Ashworth, S. (1996). La enseñanza de la EF. La reforma de los estilos de enseñanza. Barcelona: Hispano Europea.

Sánchez Bañuelos, F. (1986). Bases para una didáctica de la EF y el deporte. Madrid: Gymnos.

\section{Legislación utilizada:}

Decreto 231/2007 de 31 de julio por el que se establecen las enseñanzas correspondientes a la Educación Secundaria Obligatoria en Andalucía (BOJA 8-8-2007).

Ley Orgánica de Educación 2/2006 de 3 de mayo (BOE, 106 de 4 de mayo).

Orden 10 de Agosto de 2007 por la que se establecen las enseñanzas de Educación Primaria en Andalucía (BOJA 30-8-2007).

Orden 13 de Julio de 1994 por la que se regula el proceso de diseño, desarrollo y aplicación de adaptaciones curriculares en los centros docentes (BOJA de 10-8-1994).

Orden 17 de Enero de 1996 que establece la organización y el funcionamiento de los programas sobre Educación en Valores y Temas Transversales del currículum (BOJA de 17-2-1996)

Orden 19 de Diciembre de 1995 por el que se establece el desarrollo de la Educación en Valores en los centros docentes de Andalucía (BOJA de 20-1996).

Orden 6 de Junio de 1995 por la que se aprueba los objetivos y el funcionamiento del programa de Cultura Andaluza (BOJA de 28-6-1995).

Orden de 28 de Octubre de 1993, por la que se establecen criterios y orientaciones para la elaboración de Proyectos Curriculares de Centro y secuenciación de contenidos, así como la distribución horaria y de materias optativas en la Educación Secundaria Obligatoria. (BOJA 7-12-1993).

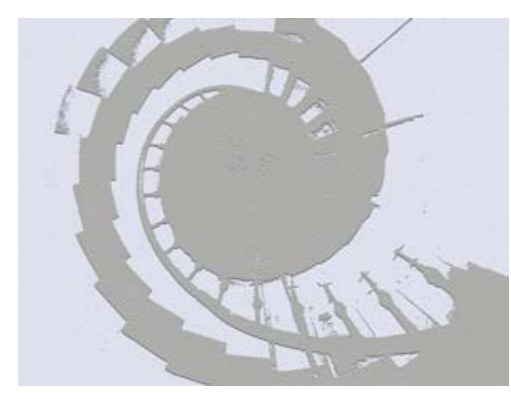

